\title{
Impact of non-pharmaceutical interventions against COVID-19 in Europe in 2020: a quasi-experimental non-equivalent group and time series design study
}

Paul R Hunter ${ }^{1,2}$, Felipe J Colón-González ${ }^{3,4,5}$, Julii Brainard ${ }^{1}$, Steven Rushton ${ }^{6}$

1. Norwich Medical School, University of East Anglia, Norwich, United Kingdom

2. Department of Environmental Health, Tshwane University of Technology, Pretoria, South Africa

3. Department of Infectious Disease Epidemiology, London School of Hygiene and Tropical Medicine, London, United Kingdom

4. School of Environmental Sciences, University of East Anglia, Norwich, United Kingdom

5. Tyndall Centre for Climate Change Research, University of East Anglia, Norwich, United Kingdom

6. School of Natural and Environmental Sciences, Newcastle University, Newcastle, United Kingdom

Correspondence: Julii Brainard (j.brainard@uea.ac.uk)

Citation style for this article:

Hunter Paul R, Colón-González Felipe J, Brainard Julii, Rushton Steven. Impact of non-pharmaceutical interventions against COVID-19 in Europe in 2020: a quasi-experimental non-equivalent group and time series design study. Euro Surveill. 2021;26(28):pii=2001401. https://doi.org/10.2807/1560-7917.

ES.2021.26.28.2001401

Article submitted on 17 Jul 2020 / accepted on 15 Mar 2021 / published on 15 July 2021

Introduction: The current pandemic of coronavirus disease (COVID-19) is unparalleled in recent history as are the social distancing interventions that have led to a considerable halt on the economic and social life of so many countries. Aim: We aimed to generate empirical evidence about which social distancing measures had the most impact in reducing case counts and mortality. Methods: We report a quasi-experimental (observational) study of the impact of various interventions for control of the outbreak through 24 April 2020. Chronological data on case numbers and deaths were taken from the daily published figures by the European Centre for Disease Prevention and Control and dates of initiation of various control strategies from the Institute of Health Metrics and Evaluation website and published sources. Our complementary analyses were modelled in $\mathrm{R}$ using Bayesian generalised additive mixed models and in STATA using multilevel mixed-effects regression models. Results: From both sets of modelling, we found that closure of education facilities, prohibiting mass gatherings and closure of some non-essential businesses were associated with reduced incidence whereas stay-at-home orders and closure of additional non-essential businesses was not associated with any independent additional impact. Conclusions: Our findings are that schools and some non-essential businesses operating 'as normal' as well as allowing mass gatherings were incompatible with suppressing disease spread. Closure of all businesses and stay at home orders are less likely to be required to keep disease incidence low. Our results help identify what were the most effective non-pharmaceutical interventions in this period.

\section{Introduction}

The current pandemic of coronavirus disease (COVID19) is unprecedented in modern history. Not only is the impact of the epidemic being measured by the number of cases and deaths, but also by its impact on overloaded health services and undesirable impacts on quality of life and near-future economic prospects. Wider society was subjected at times to an almost complete stasis of social and cultural life. The benefits of social distancing was shown earliest in China, Italy and Spain that turned the tide on their country's epidemics using often severe social distancing strategies. These examples do not indicate the relative importance of the different non-pharmaceutical/social distancing interventions. Given the potentially high economic and social costs arising from stringent control measures [1-5], it has been imperative to determine which social distancing measures are most effective at controlling the pandemic. Imposition and relaxation of control measures should be informed by such knowledge. Early on in pandemic response, much policy was driven by the results of mathematical models [6]. However, there was much concurrent public debate about the validity and limitations of the different models for policy making and modelling approaches that were used [7-10]. It is also useful to assess empirical evidence of what aspects of currently applied non-pharmaceutical interventions (NPI) have or have not been effective.

A quasi-experimental study design is an observational study where the allocation to receive the intervention (or not) is not randomly made $[11,12]$. Most European states introduced a similar suite of interventions aimed at reducing contact between individuals to reduce transmission. The different types of intervention used and their timing varied from one country to another and 


\section{FIGURE 1}

Exemplaric timeline of possible non-pharmaceutical intervention impositions and potential epidemic response, COVID-19 pandemic, Europe, 2020

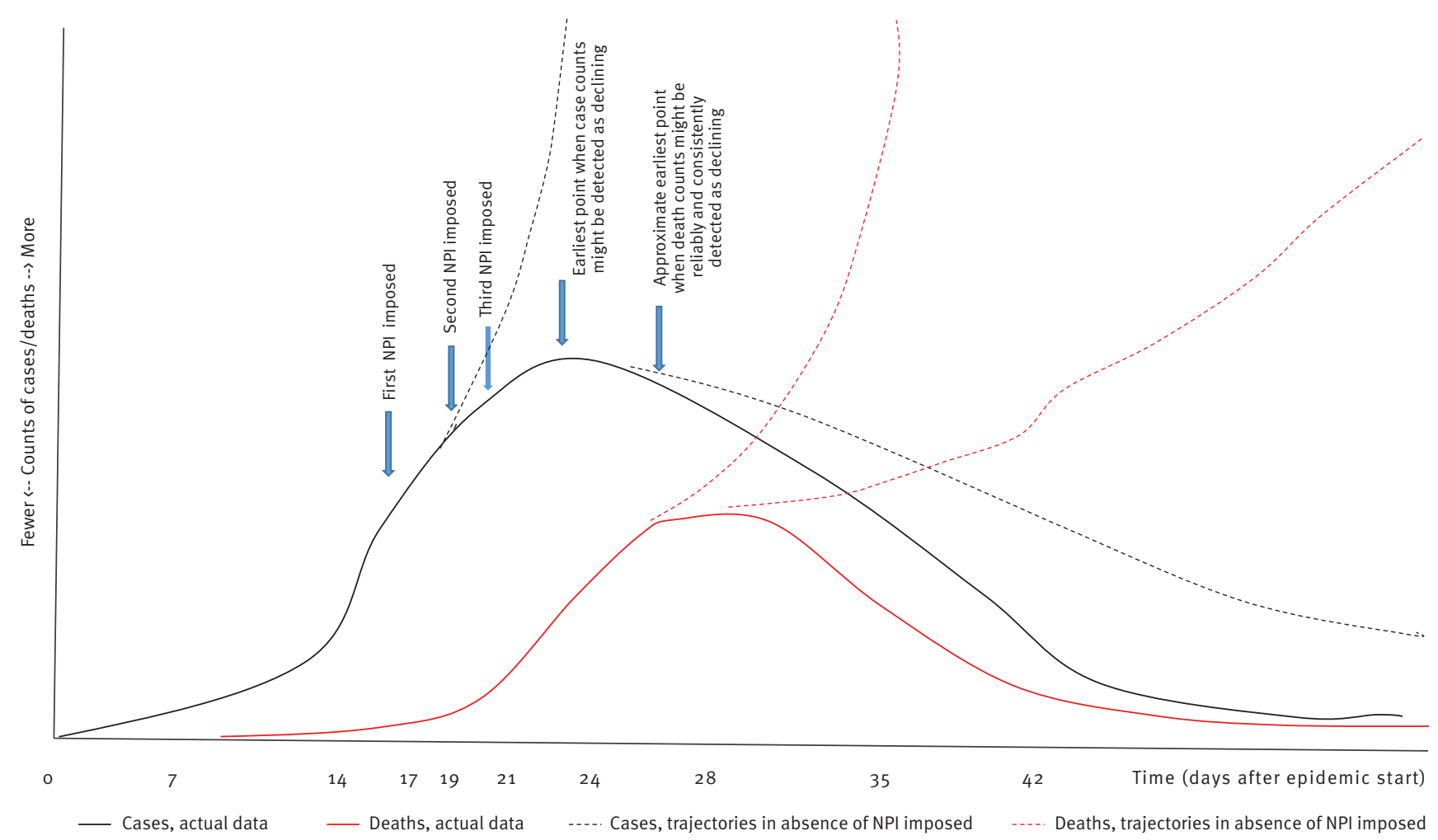

COVID-19: coronavirus disease; NPI: non-pharmaceutical intervention.

The exemplaric timeline shows how epidemic measures (counts of cases or deaths) might respond in the case of three NPI imposed. The 'earliest point' refers to the first hypothesised time point in the example, when the epidemic might have been expected to deviate from the trajectory(ies) if no NPI had been imposed.

arose in response to political processes in each country. No measure that we will consider in this analysis was imposed by all European countries. Where measures were put in place, they were often imposed at different points in the development of the epidemics. By late April 2020, some European countries were easing control measures so late April was a good point to take stock of intervention effects. This situation offered a unique opportunity to investigate the putative impacts of the various types of intervention, as each epidemic in an individual country forms what is effectively a chrono-sequence of disease spread. The intervention strategies could then be compared as interrupted time series.

We report here analyses of trends in both reported cases and deaths across 30 European countries with rather different approaches to and timing of restrictions. We use a quasi-experimental approach to identify what affects such restrictions may have had on the control of the epidemic.

\section{Methods}

Data

Data on new cases and deaths reported by all countries were obtained from the European Centre for Disease Prevention and Control (https://www.ecdc.europa. eu/en/publications-data/download-todays-data-geographic-distribution-covid-19-cases-worldwide ). Data up to 24 April 2020 are included. For the United Kingdom (UK), we used only the so-called pillar 1 case numbers. Pillar 1 refer to swab testing in Public Health England laboratories and National Health Service hospitals for those with a clinical need, and for health and care workers. Pillar 2 results (rt-PCR testing for persons with suspected COVID-19 in the wider community) as reported daily on the UK government coronavirus data website (https://www.gov.uk/guidance/coronaviruscovid-19-information-for-the-public\#history) were removed from the case numbers, as pillar 2 sampling was only introduced late in the course of the UK epidemic and inflated total case numbers relative to earlier in the UK outbreak. We also adjusted our results by the number of tests reported per 1 million population, 
TABLE 1

Timing of estimated start of each country's main COVID-19 epidemic and the introduction of social distancing measures, 30 European countries, 2020

\begin{tabular}{|c|c|c|c|c|c|c|c|}
\hline Country & $\begin{array}{l}\text { Start of } \\
\text { main } \\
\text { epidemic }\end{array}$ & $\begin{array}{l}\text { Mass gathering } \\
\text { restrictions }\end{array}$ & $\begin{array}{l}\text { Initial business } \\
\text { closure }\end{array}$ & $\begin{array}{c}\text { Educational } \\
\text { facilities closed }\end{array}$ & $\begin{array}{l}\text { Non-essential } \\
\text { services closed }\end{array}$ & $\begin{array}{l}\text { Stay at } \\
\text { home } \\
\text { order }\end{array}$ & $\begin{array}{l}\text { Face covering } \\
\text { encouraged or } \\
\text { compulsory }\end{array}$ \\
\hline Austria & 26 Feb & $10 \mathrm{Mar}$ & $16 \mathrm{Mar}$ & $16 \mathrm{Mar}$ & $16 \mathrm{Mar}$ & 16 Mar & $6 \mathrm{Apr}$ \\
\hline Belgium & 2 Mar & 13 Mar & $13 \mathrm{Mar}$ & 14 Mar & $18 \mathrm{Mar}$ & $18 \mathrm{Mar}$ & NA \\
\hline Bulgaria & $12 \mathrm{Mar}$ & $13 \mathrm{Mar}$ & 13 Mar & 13 Mar & 13 Mar & 17 Mar & $30 \mathrm{Mar}$ \\
\hline Croatia & 11 Mar & 9 Mar & 19 Mar & $16 \mathrm{Mar}$ & 19 Mar & 17 Mar & NA \\
\hline Cyprus & $10 \mathrm{Mar}$ & 24 Mar & $24 \mathrm{Mar}$ & 13 Mar & $24 \mathrm{Mar}$ & 24 Mar & NA \\
\hline Czechia & 2 Mar & $10 \mathrm{Mar}$ & $10 \mathrm{Mar}$ & $10 \mathrm{Mar}$ & 14 Mar & $16 \mathrm{Mar}$ & $18 \mathrm{Mar}$ \\
\hline Denmark & $27 \mathrm{Feb}$ & $18 \mathrm{Mar}$ & 18 Mar & $16 \mathrm{Mar}$ & NA & NA & NA \\
\hline Estonia & 11 Mar & 13 Mar & 13 Mar & 16 Mar & NA & NA & $5 \mathrm{Apr}$ \\
\hline Finland & $27 \mathrm{Feb}$ & $12 \mathrm{Mar}$ & $18 \mathrm{Mar}$ & 18 Mar & $4 \mathrm{Apr}$ & NA & NA \\
\hline France & $26 \mathrm{Feb}$ & 4 Mar & 14 Mar & $12 \mathrm{Mar}$ & 14 Mar & $16 \mathrm{Mar}$ & $5 \mathrm{Apr}$ \\
\hline Germany & $26 \mathrm{Feb}$ & 22 Mar & 17 Mar & 16 Mar & $23 \mathrm{Mar}$ & 22 Mar & $1 \mathrm{Apr}$ \\
\hline Greece & $5 \mathrm{Mar}$ & 8 Mar & $12 \mathrm{Mar}$ & 11 Mar & $22 \mathrm{Mar}$ & 23 Mar & NA \\
\hline Hungary & 5 Mar & $12 \mathrm{Mar}$ & $12 \mathrm{Mar}$ & 16 Mar & $16 \mathrm{Mar}$ & $28 \mathrm{Mar}$ & NA \\
\hline Ireland & 4 Mar & $12 \mathrm{Mar}$ & 15 Mar & $12 \mathrm{Mar}$ & 24 Mar & 27 Mar & NA \\
\hline Italy & 22 Feb & 11 Mar & $11 \mathrm{Feb}$ & 5 Mar & 11 Mar & 11 Mar & $6 \mathrm{Apr}$ \\
\hline Latvia & 8 Mar & 13 Mar & NA & $12 \mathrm{Mar}$ & NA & NA & NA \\
\hline Lithuania & 14 Mar & 15 Mar & 14 Mar & $16 \mathrm{Mar}$ & 15 Mar & 15 Mar & $1 \mathrm{Apr}$ \\
\hline Luxembourg & $7 \mathrm{Mar}$ & 13 Mar & $18 \mathrm{Mar}$ & $16 \mathrm{Mar}$ & $18 \mathrm{Mar}$ & NA & $20 \mathrm{Apr}$ \\
\hline Malta & $8 \mathrm{Mar}$ & NA & $17 \mathrm{Mar}$ & 13 Mar & 23 Mar & NA & NA \\
\hline Netherlands & $28 \mathrm{Feb}$ & $10 \mathrm{Mar}$ & $21 \mathrm{Mar}$ & 15 Mar & NA & NA & NA \\
\hline Norway & 27 Feb & 12 Mar & 12 Mar & 12 Mar & NA & NA & $5 \mathrm{Apr}$ \\
\hline Poland & $7 \mathrm{Mar}$ & $10 \mathrm{Mar}$ & 31 Mar & 12 Mar & NA & 24 Mar & NA \\
\hline Portugal & 3 Mar & 19 Mar & $16 \mathrm{Mar}$ & 16 Mar & $19 \mathrm{Mar}$ & 19 Mar & $16 \mathrm{Apr}$ \\
\hline Romania & 4 Mar & 6 Mar & 21 Mar & 11 Mar & 21 Mar & $23 \mathrm{Mar}$ & NA \\
\hline Slovakia & 7 Mar & 12 Mar & 16 Mar & 12 Mar & 16 Mar & NA & 14 Mar \\
\hline Slovenia & $5 \mathrm{Mar}$ & $12 \mathrm{Mar}$ & 15 Mar & $16 \mathrm{Mar}$ & 15 Mar & $20 \mathrm{Mar}$ & 29 Mar \\
\hline Spain & $25 \mathrm{Feb}$ & 15 Mar & $15 \mathrm{Mar}$ & 14 Mar & 15 Mar & 15 Mar & $13 \mathrm{Apr}$ \\
\hline Sweden & $27 \mathrm{Feb}$ & 11 Mar & NA & NA & NA & NA & NA \\
\hline Switzerland & $26 \mathrm{Feb}$ & $28 \mathrm{Feb}$ & $16 \mathrm{Mar}$ & 13 Mar & $16 \mathrm{Mar}$ & NA & NA \\
\hline United Kingdom & $28 \mathrm{Feb}$ & 23 Mar & $20 \mathrm{Mar}$ & 23 Mar & 24 Mar & $23 \mathrm{Mar}$ & NA \\
\hline
\end{tabular}

NA: not applicable, as this control was not implemented; COVID-19: coronavirus disease.

taken on 16 April from WorldoMeter (https://www.worldometers.info/coronavirus/). In order to compare time series for different countries with different dates of onset for their own epidemics we chose to define the onset as the first day when a case was reported after the latest time where there were two or more consecutive days with no cases reported.

The dates when (if at all) each of the various social restrictions were imposed in the 30 European countries included in this analysis were given by the Institute of Health Metrics and Evaluation Data (IHME) (https://covid19.healthdata.org/). The six categories of restrictions were 'mass gathering restrictions', 'initial business closure', 'educational facilities closed', 'non-essential services closed', 'stay-at-home order' and 'travel severely limited'. However, no country was listed in the dataset as having severe travel restrictions during the monitoring period so we dropped this category from any further analysis. The IHME definitions of these measures are given on their website. We paraphrase the definitions here:

- Mass gathering restrictions were mandatory restrictions on private or public gatherings of any number of people.

- Initial business closure refers to the first time that there was any mandatory closure of businesses, not necessarily all businesses. Usually such initial closures would primarily affect businesses such as entertainment venues, bars and restaurants.

- Where non-essential businesses were ordered to close, this usually included many more businesses than were in the first closure category. The second wave of closures probably included general retail stores and services such as hairdressers. 
TABLE 2

Model metrics, impact of non-pharmaceutical interventions, COVID-19 pandemic, Europe, 2020

\begin{tabular}{|c|c|c|c|c|}
\hline Model & Deviance information criterion & Watanabe-Akaike information criterion & Conditional predictive ordinate & Dispersion \\
\hline Cases & $18,009.4$ & $18,012.6$ & $-9,006.6$ & 1.01 \\
\hline Deaths & $8,032.4$ & $8,035.9$ & $-4,018.4$ & 0.89 \\
\hline
\end{tabular}

COVID-19: coronavirus disease.

The Watanabe-Akaike information criterion (W-AIC) is described by Watanabe in 2010 [46] and was developed to specifically help identify best model fit in Bayesian models. Smaller W-AIC values mean better fit compared with alternative model specifications. The conditional predictive ordinate is a Bayesian diagnostic that detects surprising observations [47].

- Closure of education facilities included all levels of education (primary, secondary and higher) that stopped face-to-face teacher-to-student teaching.

- Stay-at-home orders affected all individuals unless travelling for essential services. They allowed close contact only with people of the same household and perhaps some outdoors exercise.

In three countries (Germany, Italy and Spain), the restrictions were not implemented uniformly through the country on precisely the same dates so we took the median date for the nation; the actual variations in dates were extremely small in Italy and Spain and only somewhat diverse in Germany (see Supplement 1, part 1). Among the 16 German states, 15 states imposed mass gathering restrictions within 2 days of the median date used, nine states had initial business closures within 2 days of the median date, 15 states closed educational establishments within 2 days of the median date, nine states closed non-essential businesses within 2 days of the median German date and all states imposed stay-at-home orders within 2 days of the median national date.

All models adjusted for when countries started to advise or mandate their citizens to wear face masks or coverings (dates of face cover measures are listed in Supplement 1, part 2). We included when countries either mandated or encouraged the wearing of face coverings or masks in public places as an independent control measure in the models. However, it was obvious that how such advisories or mandates were implemented varied considerably from one country to another. For example, in some countries, face masks were required both outdoors and indoors in public and in others only in indoor settings. Sometimes, mask wearing was required in few settings such as on public transport, other times in several settings such as on public transport, in shops and in schools. Also mask-wearing mandates, where implemented, were only introduced relatively late in the monitoring period, often even as other control measures were being relaxed, which complicated interpretation of how much masks may have helped reduce transmission. Consequently, although we included the wearing of face coverings in the analyses, we caution against drawing any strong conclusions over their value based on these analyses alone.

\section{Analyses}

We undertook two sets of analyses. In order to ensure comparability between countries with different timing of their outbreaks we counted dates as being from the start, the epidemic in each country was assumed to have commenced on the first day with a reported case after the last time that no cases were reported on two consecutive days.

The first analysis was done in $\mathrm{R}$ using Bayesian generalised additive mixed-effects models. These incorporate both fixed and random effects (i.e. mixed effects) to adjust for spatial dependency in disease between nation states. Random effects correspond to those for which levels are samples from a larger population, whereas fixed effects correspond to average effects for the whole population. Examples of fixed effects would be interventions such as shutting all schools and making people work at home. Other sources of variation that contribute may be more random and associated with unmeasured features of the sampling unit (the nation state). Key here is the fact that the nation states differ culturally and in other features such as recording methods. We have not measured the source of the variation but we know it is associated with the sampling unit (state) with which the response is recorded through time. In addition, we also anticipated spatial effects because most European states experiencing COVID-19 epidemics had porous land borders under the Schengen Area agreement. We therefore expect some spatial dependency between states as the closer they are to each other the more likely it is that they have similar patterns of disease. Bayesian models are very useful as they allow us to quantify the relative contributions of fixed, random, temporal and spatial dependency in the same modelling framework.

The variance in the COVID-19 data was four orders of magnitude larger than the mean number of cases and three orders of magnitude larger than the mean number of deaths. Consequently, models were fit using a negative binomial specification to account for potential over-dispersion in the data, and within a conditional autoregressive model (Besag-York-Mollié) framework [13] to allow for potential spatial autocorrelation and unstructured between-country variation. 


\section{FIGURE 2}

Incidence rate ratios (cases) following implementation of country-level, non-pharmaceutical control measures and daily reported COVID-19 case numbers, 30 European countries, 2020

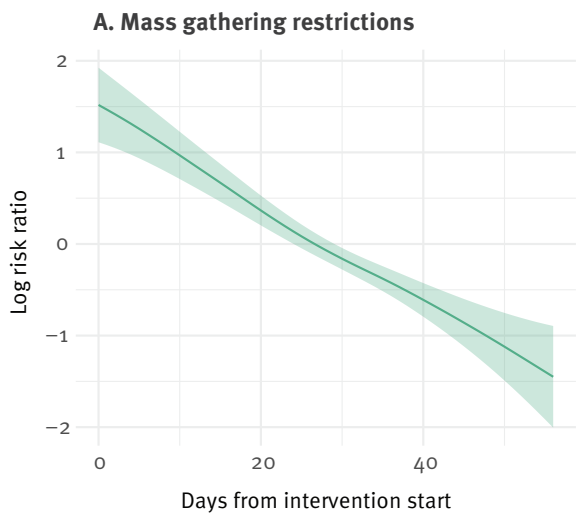

D. Non-essential business closure

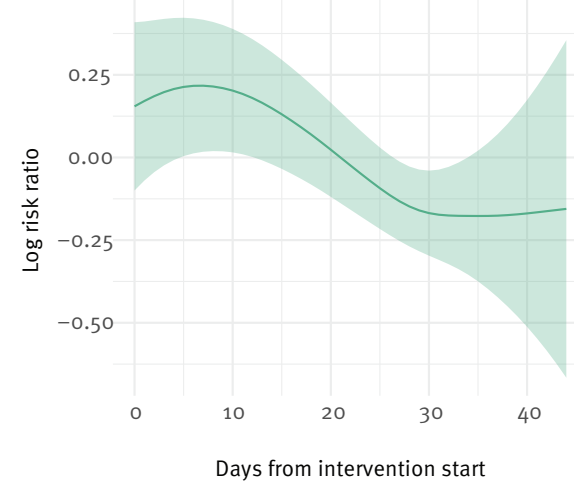

B. Initial business closure

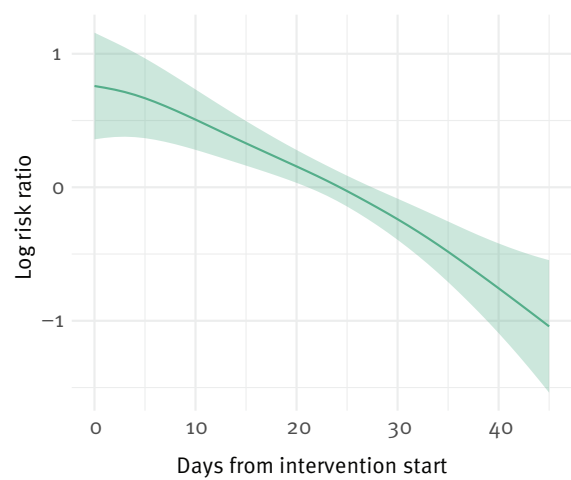

E. Stay home measures

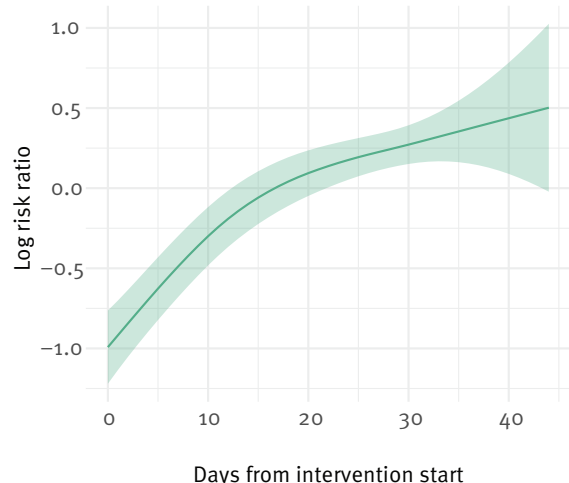

C. Education facilities closure

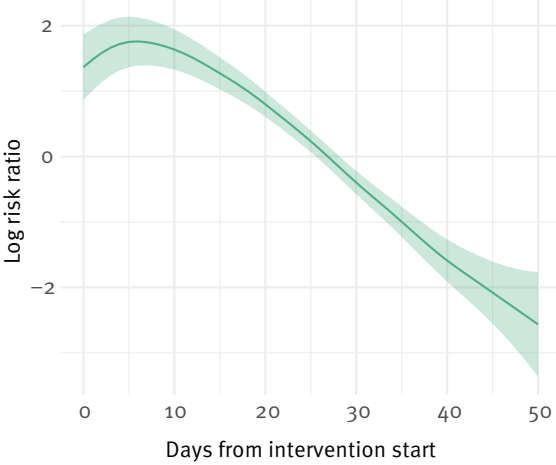

F. Mask usage

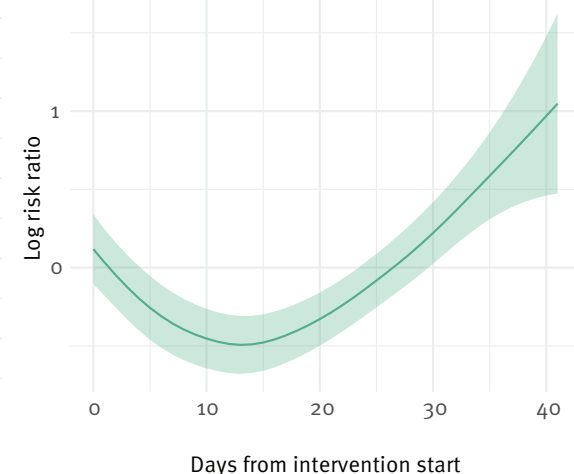

COVID-19: coronavirus disease.

The central line is the posterior mean of the exposure-response relationship; shading is a $95 \%$ credible interval.

Let $\mathrm{Yi}, t$ be the number of COVID-19 cases or deaths for country $i=1, \cdots, l$ at time $t=1, \cdots, T$. The general algebraic definition of the models is given by:

$$
Y_{i, t} \mid \mu_{i, t}, \phi \sim \operatorname{NegBin}\left(\mu_{i, t}, \phi\right),
$$

where $Y_{i, t}$ is the number of COVID-19 cases or deaths for country $i=1, \cdots, l$ at time $t=1, \cdots, \mu_{i, t}$ is the predicted number of COVID-19 cases or deaths for country $i$ and time $t$, and $\phi>0$ is the negative binomial dispersion parameter. A logarithmic link function of the expected number of cases or deaths was modelled as:

$\log (\mu \mathrm{i}, \mathrm{t})=\alpha+\log (\mathrm{Pi}, \mathrm{d}[\mathrm{t}])+\delta \mathrm{Di}, \mathrm{d}[\mathrm{t}]+\epsilon \mathrm{Ri}, \mathrm{d}[\mathrm{t}]+\sum_{k} \beta X_{i, t, k}+\mathrm{ui}+\nu \mathrm{i}$,

where a corresponded to the intercept; $\log \left(P_{i, d[t]}\right)$ denotes the logarithm of the population at risk for country $i$ and day $d_{[t]}$ was included as an offset to adjust case counts by population. $D_{i, d[t]}$ is a linear term for the number of days since the outbreak started, with coefficient $\delta . R_{i, d[t]}$ is a linear function of the number of COVID-19 tests carried out per country $i$ at day $d_{[t]}$, with regression coefficient $\epsilon . X$ is a matrix of $k$ intervention measures (e.g. school and business closures) with regression coefficients $\beta$. Intervention measures comprise of an index of $1, \cdots, N$ number of days following the intervention being implemented (day 1 was the day following implementation of the intervention). We assumed that the imposition of each intervention led to cumulative changes in effect. Intervention measures were included in the model as a random effect to account for potential nonlinearities in the exposure-response relationship. A random effect adjustment was appropriate because the observation data (case counts) were samples from a larger population (because of limited testing to confirm symptomatic cases and possible asymptomatic cases). Unknown confounding factors with spatial dependency that represent, for example, human mobility, were incorporated using spatially correlated (i.e. structured) random effects $\left(u_{i}\right)$ and independent, identical and normal distributed (i.e. unstructured) random effects $\left(v_{i}\right)$ for each country $i$. Spatial random effects were specified using a Besag-York-Mollie model to account for spatial dependencies and unstructured variation between countries [14]. Goodness of fit was evaluated 


\section{FIGURE 3}

Incidence rate ratios (deaths) following implementation of country-level, non-pharmaceutical control measures and daily reported deaths from COVID-19, 30 European countries, 2020
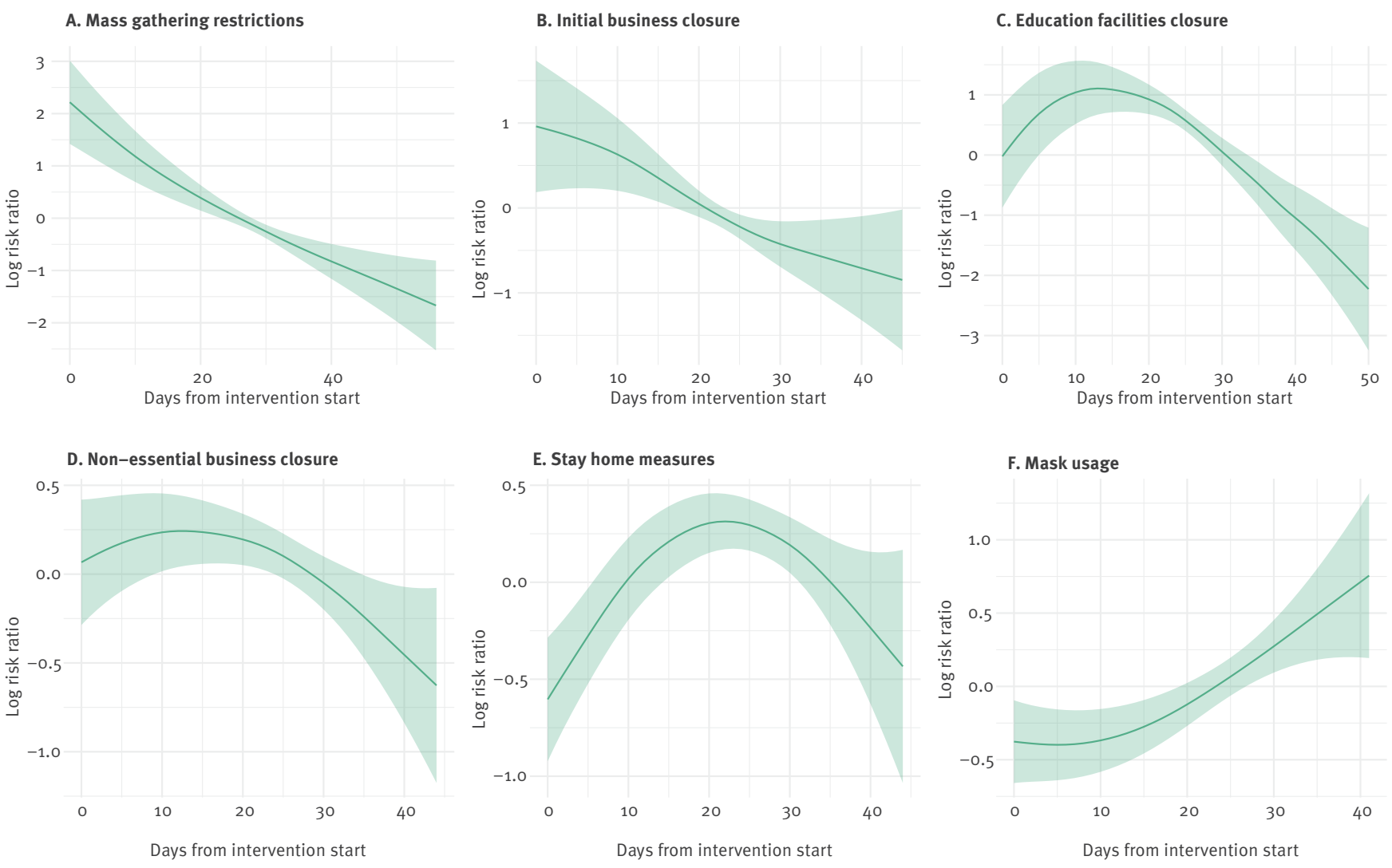

COVID-19: coronavirus disease.

The central line is the posterior mean of the exposure-response relationship; shading is a $95 \%$ credible interval.

using the deviance information criterion (DIC). Models were fitted in $\mathrm{R}$ version 3.6.1 using the INLA package.

The second analysis was a multilevel mixed-effects regression analysis in STATA $\vee 16.1$ (StataCorp LLC, College Station, United States). We used a mixedeffects negative binomial regression model with cases or deaths on a specific day as the outcome variable, country population as the exposure variable, country as a mixed effect and days from start of the epidemic as a fixed effect. Fixed effect was appropriate for days elapsed because we were looking for possible effect of NPI relevant to a fixed start point and over the entire population. All main interventions were included as categorical variables with the week number included as a linear variable after the start of the intervention. Monitoring by week number was appropriate with regard to case counts, given that the incubation period tends to be ca 5 days [15-18] and a small lag between symptom onset and obtaining test results is likely: thus, total days elapsed from exposure to changes in recorded case counts has tended to be ca 7 days. A lag from symptom onset to hospitalisation of ca 7 days $[19,20]$ and a similar subsequent lag (ca 7 days) from hospitalisation to death are reported in COVID19 literature [19-21]. Figure 1 indicates the impact of key likely onsets of intervention on an exemplaric epidemic curve. For simplicity and brevity we report only on the results for the 7 -day categorisation in this manuscript. However, in view of the variation in incubation period and the possibility that this might have interfered with the parameter estimates, we repeated Analysis 2 for three alternative response time periods (post-intervention) as sensitivity analyses. These alternative response periods were 4 days, 10 days and 14 days. The resulting incident risk ratios (between our preferred response period of 7 days and alternatives) could then be compared for possible trend differences. In further sensitivity and collinearity checks, we dropped each of the main predictor variables (intervention timings) from the final equation and noted if the regression parameter and standard errors of remaining predictor variables changed dramatically or if the coefficients reversed trend (e.g. went from suggesting increase to suggesting decrease).

We also checked for collinearity between the predictor variables by calculating the variance inflation factors 


\section{FIGURE 4}

Comparison of predicted daily reports of COVID-19 case numbers with 7-day rolling average actual numbers, 30 European countries, 2020
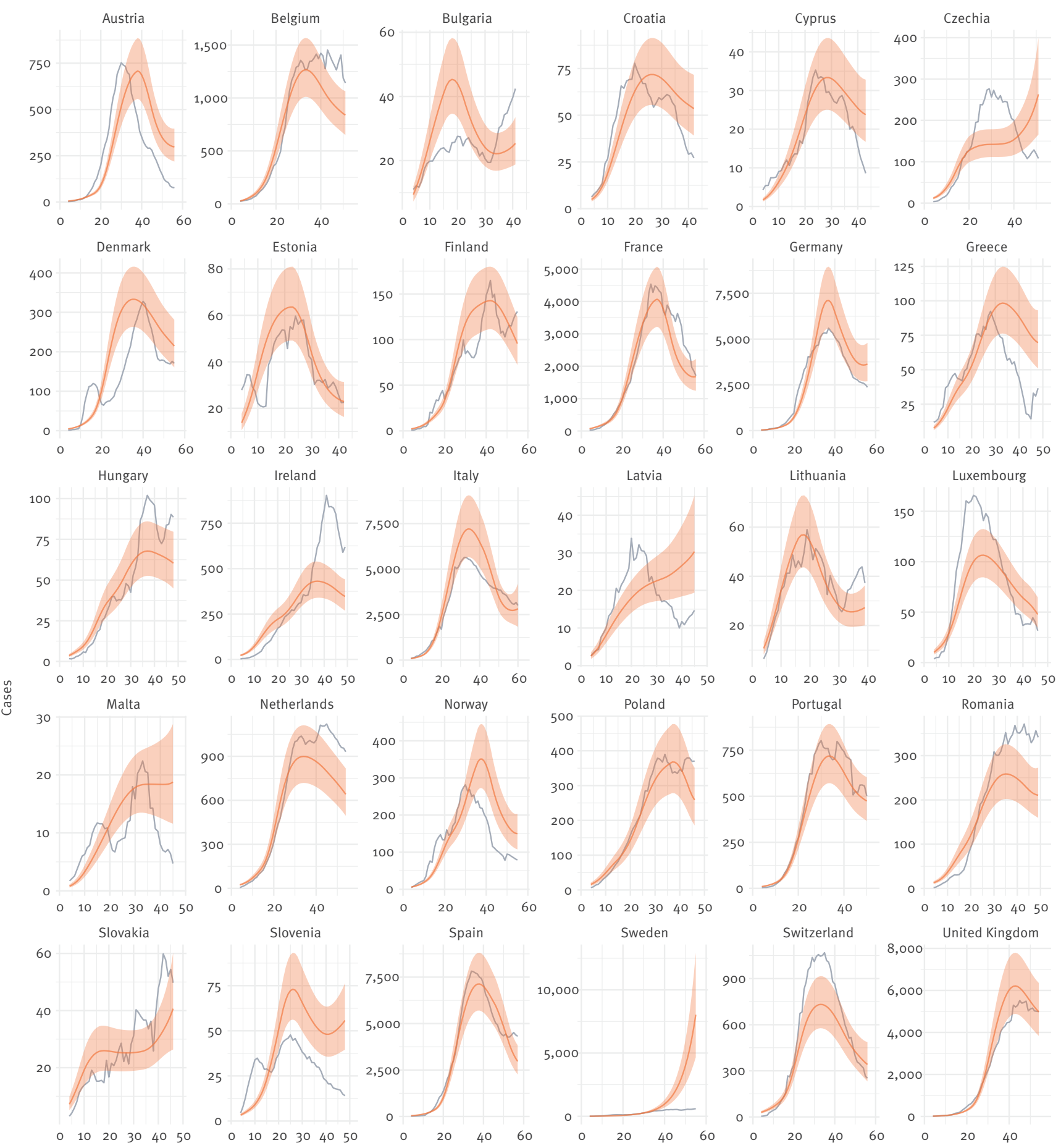

first day of epidemic

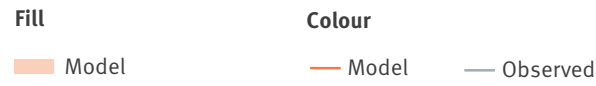

COVID-19: coronavirus disease.

The central line is the posterior mean of the predictions made by the models (for individual countries over time); shading is the $95 \%$ credible interval. 
(VIF) for the predictors and by calculating the condition number using the coldiag 2 command in STATA. A VIF $<10$ suggests that model predictors do not have multi-collinearity problems. Values of VIF $>10.0$ need to be considered for potential multi-collinearity with regard to other model diagnostics such as condition index and eigenvalues. A condition number $>15$ with any variance proportions above 0.9 , or if eigenvalues were $<0.01$, could suggest collinearity that undermines confidence in coefficient estimates, according to guidance in Chatterjee and Hadi [22] and Regorz [23]. In addition, as sensitivity analysis within Analysis 2, we reran the model dropping each predictor variable in turn to determine whether or not the regression parameters and their standard errors were changed substantially.

\section{Ethical statement}

Ethical approval was not required because this was an analysis of data in the public domain.

\section{Results}

Table 1 lists the estimated date of the start of the epidemic in each country and when each of the five intervention types were implemented, according to the IHME website. 'Mass gathering restrictions', 'initial business closure', 'educational facilities closed', 'nonessential services closed' and 'stay at home order' were respectively implemented by $29,28,29,23$ and 19 countries. Italy was the first country to enter the epidemic on 22 February 2020 and Lithuania the last on 14 March 2020. By our criteria, half of all countries had their epidemic start on or before 27 February.

\section{Analysis 1}

Model metrics are presented in Table 2. The dispersion parameter evaluates whether the model is able to cope with potential dispersion in the data. When the value is close to 1 (as it is here) the model is shown to do well at accounting for dispersion.

The exposure-response relationships estimated by the models are presented in Figures 2 (cases) and Figure 3 (deaths). The $x$-axes represent the days since the intervention started and the $y$-axes indicate the logarithm of the risk ratio. It can be observed that mass gathering restrictions had a negative effect on the number of cases, with fewer cases occurring as the number of days since intervention started increased. We observed a similar effect for the initial closure of businesses and the closure of education facilities, with less cases occurring as the number of days since the intervention increased. The closure of non-essential businesses did not appear to have a significant effect on the number of COVID-19 cases. This was evident as the estimated relationship and its $95 \%$ credible interval stayed close to zero on the y-axis. Surprisingly, stayat-home measures showed a positive association with cases. This suggests that, as the number of lockdown days increased, so did the number of cases. Negative associations with deaths (Figure 3) were estimated for mass gatherings, initial business closure and the closure of educational facilities, while a non-significant effect was estimated for non-essential business closure. The stay-at-home measures showed an inverted U-quadratic effect with an initial rise of deaths up to Day 20 of the intervention, followed by a decrease. These results suggest that stay-at-home orders may not be required to ensure outbreak control and reduce outbreak harms, provided that all the other control measures are implemented. Of course, if stay-at-home measures are implemented then all the other measures such as business closures, banning mass gatherings and school closures would also follow.

The patterns seen in Figures 2 and 3 fit with the understood disease incubation, development and concurrent ascertainment processes. The median incubation period is understood to be 4-7 days [15-17], while case ascertainment tended to require an elapse of 2-10 more days [24]. For severe cases (those who are hospitalised), 8-14 days post symptom onset tends to coincide with the start of a 5-7-day period of peak disease severity [20]. As a result, we expect no intervention should be cited as affecting case counts in under about 7 days, and no intervention is likely to strongly reduce counts of death in less than $2-3$ weeks.

For cases and deaths, mask wearing mandates/advisories seem to have initial effects which were either negative (case) or neutral (deaths), followed by rises (in cases or deaths). The overall effect is small compared to other measures, which we confirmed with further sensitivity analyses shown below. The additional benefit of mask-wearing advisories/mandates to the other outbreak control measures seemed to be small and inconsistent. However, for the reasons discussed above we hesitate to interpret these results as certain effects of face cover/mask mandates/advisories.

Figures 4 and 5 show the association between actual cases and deaths in each country, expressed as 7-day rolling means, and the numbers predicted by the models on cases and deaths. Although for many countries there is a reasonable correlation between the two, this is not the case for all countries and particularly countries with smaller populations. The model outputs especially did not fit Sweden which had much lower numbers of cases and deaths than predicted. This could be explained by partial implementation of controls and unmandated behavioural change in the population. We acknowledge that, at least for some countries, our model could not capture all the temporally changing variables influencing the spread of the disease.

Figure 6 shows the maps of the posterior mean for the country-specific relative risks of COVID-19 cases (panel A) and COVID-19 deaths (panel C). These country-specific risks enable comparison of individual countries to case/death incidence in the whole study area, having accounted for the effects of all other covariates in the model. Figures $6 \mathrm{~A}$ and $6 \mathrm{C}$ indicate whether the cases or deaths per 100,000 were higher or 
Comparison of predicted daily numbers of reports of deaths from COVID-19 with 7-day rolling average actual numbers, 30 European countries, 2020
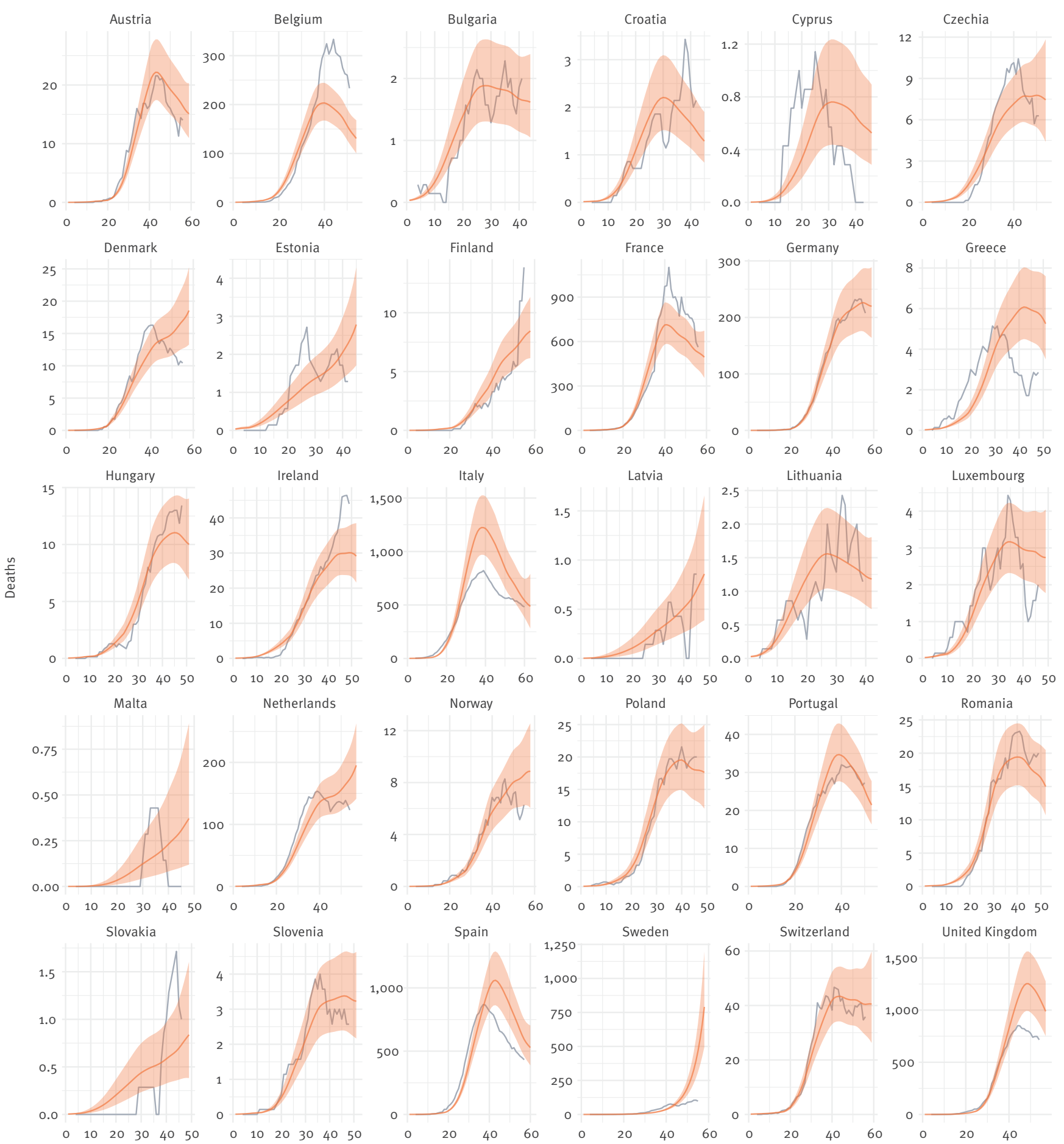

first day of epidemic

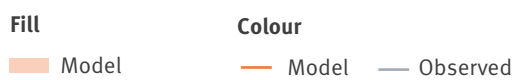

COVID-19: coronavirus disease.

The central line is the posterior mean of the predictions made by the models (for individual countries over time); shading is the $95 \%$ credible interval. 
Posterior mean of the country-specific risk ratio of COVID-19 cases and deaths and posterior probability of exceeding one COVID-19 case or death, 30 European countries, 2020

\section{A. Risk ratio COVID-19 cases}

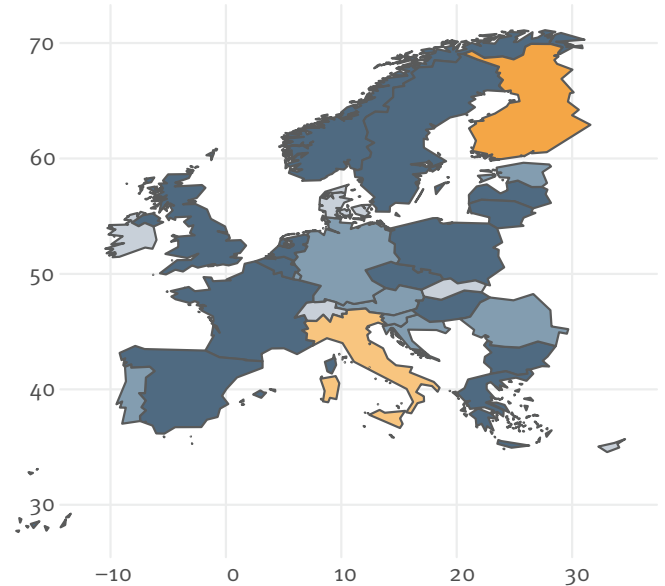

C. Risk ratio COVID-19 deaths

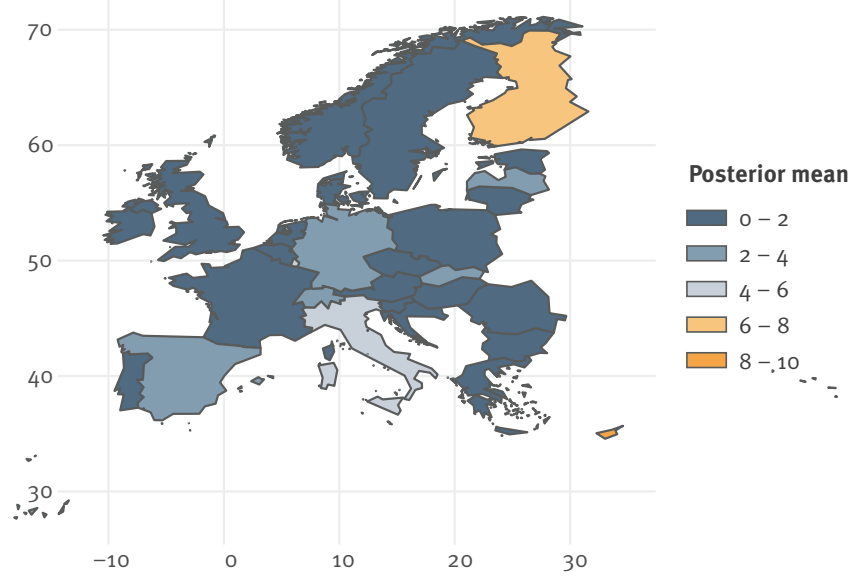

B. Posterior probability of exceeding one COVID-19 case

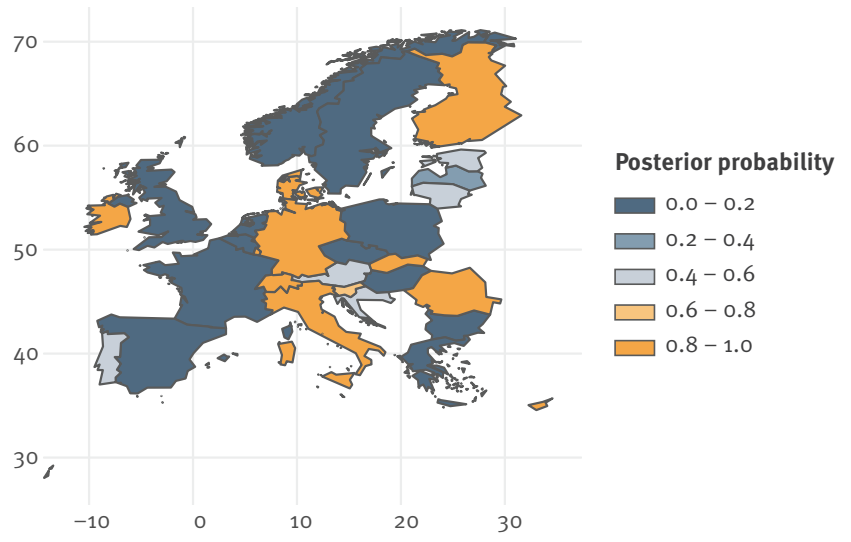

D. Posterior probability of exceeding one COVID-19 death

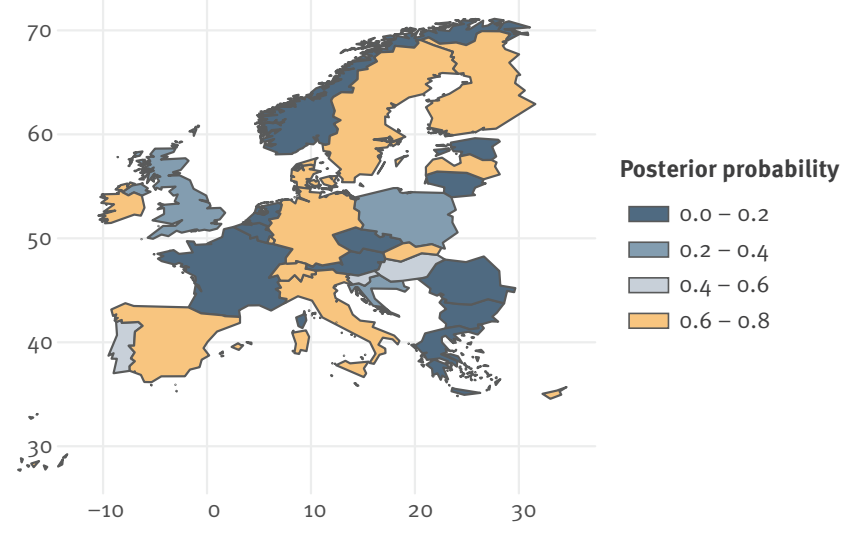

COVID-19: coronavirus disease.

Country-specific relative risks compared with the whole of the study area have accounted for the effects of all other covariates in the model; posterior probability of exceeding one case or death is calculated per 100,000 persons after adjusting for covariates.

lower in a given country relative to the incidence in the full region. Posterior means in the top two categories (shades of orange) indicate especially high excess of country-specific risk relative to cases/deaths in the whole region. Posterior means lower than 1.0 (dark blue) indicate a lower risk ratio than that of the whole region. Figures $6 \mathrm{~B}$ and $6 \mathrm{D}$ show the country-specific posterior probability (range: $0-1$ ) of observing a relative risk larger than one compared with case/death incidence in all 30 countries. The proportion of spatial variance explained by the models is $16 \%$ for the casespecific model and $15 \%$ for the death-specific model. These values $(15-16 \%)$ are not high, indicating that the spatial components of the models are not highly explanatory of the variability in cases/deaths.

\section{Analysis 2}

For confirmation and comparison, we repeated the analysis using a multilevel mixed-effects model with results shown in Table 3. The conclusions of this analysis were broadly the same as for the hierarchical probabilistic models described above. The coefficients for these models assesses the independent contributions of the interventions to the outcomes while holding the others at their mean (as we would expect from a multivariate linear model). The incident risk ratios (IRR) are shown in Table 3 with 95\% credible intervals, for either deaths or cases, for each period (each week) after the intervention started. Larger IRR values suggest greater effects; a value of 1 implies no effect, values above 1.0 suggest increase in cases/deaths, while values below 1 imply decrease. For time periods $1-7$ and 8-14 


\section{TABLE 3}

Results of mixed-effects negative binomial model of the effect of each intervention on COVID-19 case numbers and deaths, 30 European countries, 2020

\begin{tabular}{|c|c|c|c|c|c|}
\hline \multirow{2}{*}{ Intervention } & \multirow{2}{*}{ Timing } & \multicolumn{2}{|c|}{ Cases } & \multicolumn{2}{|c|}{ Deaths } \\
\hline & & IRR & $95 \% \mathrm{Cl}$ & IRR & $95 \% \mathrm{Cl}$ \\
\hline \multirow{7}{*}{ Mass gathering restrictions } & Before & 1 & Reference & 1 & Reference \\
\hline & 1-7 days after & 1.32 & $1.10-1.57$ & 0.76 & $0.55-1.03$ \\
\hline & $8-14$ days after & 1.13 & $0.88-1.43$ & 0.58 & $0.41-0.84$ \\
\hline & $15-21$ days after & 0.99 & $0.73-1.34$ & 0.59 & $0.38-0.92$ \\
\hline & 22-28 days after & 0.80 & $0.56-1.15$ & 0.56 & $0.33-0.93$ \\
\hline & $29-35$ days after & 0.74 & $0.48-1.13$ & 0.50 & $0.28-0.91$ \\
\hline & $\geq 36$ days after & 0.66 & $0.40-1.09$ & 0.49 & $0.25-0.98$ \\
\hline \multirow{7}{*}{ Initial business closures } & Before & 1 & Reference & 1 & Reference \\
\hline & 1-7 days after & 1.18 & $0.96-1.46$ & 1.07 & $0.80-1.43$ \\
\hline & $8-14$ days after & 0.87 & $0.66-1.15$ & 1.07 & $0.75-1.54$ \\
\hline & $15-21$ days after & 0.69 & $0.49-0.96$ & 0.72 & $0.47-1.11$ \\
\hline & 22-28 days after & 0.61 & $0.41-0.91$ & 0.50 & $0.29-0.83$ \\
\hline & $29-35$ days after & 0.47 & $0.29-0.76$ & 0.42 & $0.22-0.77$ \\
\hline & $\geq 36$ days after & 0.32 & $0.18-0.56$ & 0.37 & $0.18-0.77$ \\
\hline \multirow{7}{*}{ Educational facilities closed } & Before & 1 & Reference & 1 & Reference \\
\hline & 1-7 days after & 1.47 & $1.22-1.79$ & 2.51 & $1.89-3.34$ \\
\hline & $8-14$ days after & 1.38 & $1.05-1.80$ & 3.14 & $2.14-4.62$ \\
\hline & $15-21$ days after & 0.95 & $0.67-1.33$ & 2.76 & $1.74-4.36$ \\
\hline & 22-28 days after & 0.52 & $0.35-0.78$ & 2.02 & $1.19-3.43$ \\
\hline & 29-35 days after & 0.26 & $0.16-0.42$ & 1.10 & $0.60-2.01$ \\
\hline & $\geq 36$ days after & 0.14 & $0.08-0.25$ & 0.55 & $0.28-1.10$ \\
\hline \multirow{7}{*}{ Non-essential services closed } & Before & 1 & Reference & 1 & Reference \\
\hline & 1-7 days after & 1.14 & $0.92-1.41$ & 1.40 & $1.03-1.90$ \\
\hline & $8-14$ days after & 1.15 & $0.90-1.47$ & 1.41 & $1.00-1.97$ \\
\hline & $15-21$ days after & 1.02 & $0.78-1.33$ & 1.42 & $0.99-2.03$ \\
\hline & 22-28 days after & 0.83 & $0.60-1.13$ & 1.44 & $0.95-2.17$ \\
\hline & $29-35$ days after & 0.76 & $0.52-1.10$ & 1.04 & $0.65-1.68$ \\
\hline & $\geq 36$ days after & 0.76 & $0.46-1.26$ & 0.77 & $0.42-1.39$ \\
\hline \multirow{7}{*}{ Stay-at-home order/advisory } & Before & 1 & Reference & 1 & Reference \\
\hline & 1-7 days after & 1.19 & $0.97-1.47$ & 1.30 & $0.96-1.76$ \\
\hline & $8-14$ days after & 1.95 & $1.56-2.44$ & 2.01 & $1.45-2.77$ \\
\hline & $15-21$ days after & 2.28 & $1.79-2.90$ & 2.23 & $1.58-3.14$ \\
\hline & 22-28 days after & 2.55 & $1.94-3.35$ & 1.99 & $1.36-2.89$ \\
\hline & $29-35$ days after & 2.49 & $1.78-3.48$ & 1.84 & $1.19-2.83$ \\
\hline & $\geq 36$ days after & 2.39 & $1.49-3.84$ & 1.21 & $0.70-2.10$ \\
\hline \multirow{7}{*}{ Mask order/advisories } & Before & 1 & Reference & 1 & Reference \\
\hline & 1-7 days after & 0.66 & $0.55-0.79$ & 0.91 & $0.75-1.11$ \\
\hline & $8-14$ days after & 0.53 & $0.43-0.65$ & 0.89 & $0.71-1.12$ \\
\hline & $15-21$ days after & 0.52 & $0.40-0.67$ & 0.97 & $0.73-1.29$ \\
\hline & $22-28$ days after & 0.68 & $0.48-0.98$ & 1.40 & $0.91-2.15$ \\
\hline & $29-35$ days after & 1.15 & $0.70-1.87$ & 1.36 & $0.72-2.55$ \\
\hline & $\geq 36$ days after & 1.06 & $0.56-2.01$ & 1.45 & $0.60-3.54$ \\
\hline Days from epidemic start & Per day & 1.14 & $1.12-1.15$ & 1.17 & $1.15-1.19$ \\
\hline \multicolumn{2}{|c|}{ Tests per 1,000 population done by 16 April 2020} & 1.06 & $1.04-1.07$ & 1.02 & $0.99-0.06$ \\
\hline \multicolumn{6}{|c|}{ Random effects } \\
\hline \multicolumn{2}{|l|}{ Country (variance) } & 0.26 & $0.15-0.46$ & 1.19 & $0.70-2.03$ \\
\hline
\end{tabular}

$\mathrm{Cl}$ : confidence interval; COVID-19: coronavirus disease; IRR: incident risk ratio.

The IRR is generated by exponentiating the results of the model's raw outputs which were generated in a default log scale. 
days, the IRR values were above 1 , indicating a positive association between cases/death and the intervention variable. For periods starting 15 days onwards the IRR was generally below 1 suggesting a negative association between the outcome and the intervention. This pattern probably reflects the time lag between exposure, latency and disease detection, so that the impact of interventions only kicks in after what is effectively a lag period of 14 days. Closing schools, banning mass gatherings and initial business closures reduced cases and deaths most. Other measures had smaller and less consistent effects.

In addition, we looked at the impact of removing one intervention at a time or all interventions on the model log likelihoods (Table 4). The biggest impact came from removing educational closures from the model. The next biggest change came from removal of stay-athome orders, but this intervention was associated with a smaller decline in epidemic risk (deaths). We note that removing mask wearing as a control measure had a moderate effect on case counts but very minor effect in mortality outcomes; this difference may reflect the relatively late imposition of mask-wearing mandates/ advisories.

\section{Collinearity and sensitivity analyses}

Regression diagnostics for the alternative specifications of response time periods in Analysis 2 (4, 10 or 14 rather than 7 days) are shown in Supplement 2, with visual comparisons available in Supplement 1, part 6. There was little difference in the overall rate of decline in risk ratio with increased time since intervention regardless of time unit used. There were noticeable outliers in a few model IRR values at the longest time periods (more than 40 or 50 days) when data contributions tended to be from just one or two countries (see Supplement 1, part 6 and Supplement 2).

The VIF values for the predictor variables in Analysis 1 were all smaller that 10 (mean VIF 5.7) except for initial business closures which gave a VIF of 10.4 (Supplement 1, part 3). Collinearity diagnostics for Analysis 2 were almost identical, in that the VIF only just exceed the 10.0 threshold and only for the initial business closures variable (Supplement 1, part 4). The condition index exceeded 15.0 in the ninth dimension and suggested some collinearity between initial and non-essential business closure parameters. However, corresponding variance proportions in all dimensions for each control measure were well below 0.9. The smallest eigenvalue (Supplement 1, part 4) was 0.059 , which is above the suggested threshold of 0.01 . These tests as a group indicate that collinearity between predictor variables did not harmfully bias the apparent separate contributions of each disease control measure (as indicated by coefficient central estimates) in our models. In addition, the standard errors of the predictors in both models were small (rarely >0.20), while in the sensitivity and collinearity checks, dropping any one of the main predictor variables from the final equation of Analysis
2 did not strongly change the coefficients and standard errors of remaining predictor variables. We conclude that there was some collinearity in our models, notably between the business closure variables, but that this was not enough to affect our conclusions.

\section{Discussion}

Our analyses confirm that the imposition of non-pharmaceutical control measures have been effective in controlling epidemics in each investigated country. However, we were unable to demonstrate a strong impact from every intervention. Closure of educational facilities, banning mass gatherings and early closure of some but not necessarily all commercial businesses were all associated with reduction of the spread of infection. Widespread closure of all non-essential businesses and stay-at-home orders seem not to have had much additional value. Other analyses of actual intervention impositions and subsequent case/death counts have also found that school closures were especially effective control measures for reducing spread of COVID-19 [25-28]. However, it is vital that we caveat this finding (about closing educational establishments) by noting that it relates to closing schools that operated 'as normal' rather than when they operated with COVID-19-secure policies. We also do not attempt here to discuss what the best COVID-19 mitigation measures might be within schools.

It seems likely that many possible combinations of physical distancing measures can be effective. The apparent effects of the measures as described here may be biased by the measures themselves tending to have a sequence in common among all countries. Measures imposed later may seem less effective simply because of the order in which they happened (additional benefits were small after other measures were put in place). Other analysts have drawn this same conclusion about coronavirus NPI [28]. Our analyses indicated that school closures and stopping mass gatherings were most effective, but we acknowledge that these measures were among the earliest taken in Europe; the data did not allow us to see what marginal gains might have been achieved if school closures had been the last of all measures taken. Also, different measures reinforced and enabled each other: for instance, there was little incentive to leave home if schools and businesses were already closed and weather was inclement (as it often is in early spring in Europe, when most physical contact restrictions started). Business and school closures usually preceded stay-at-home measures in Europe, so it may not have been possible for data on stay-athome orders to be linked to large additional effects. This potential ordering problem is at least somewhat mitigated for by our use of individual lag measures (in timing) from when each intervention was effected. It is also worth noting that outside of institutional and crowded settings, there is evidence that much, if not most, COVID-19 transmission was within households in this period [29]; stay-at-home orders intensify contact within households which would be expected to 


\section{TABLE 4}

Log likelihood of each model for full model compared with models excluding each of the COVID-19 interventions and all interventions, 30 European countries, 2020

\begin{tabular}{|l|c|c|}
\hline Model & Log likelihood & Change \\
\hline Full model (cases) & $-9,081$ & NA \\
\hline Excluded \\
\hline Mass gathering restrictions & $-9,096$ & -15 \\
\hline Initial business closures & $-9,097$ & -16 \\
\hline Educational facilities closed & $-9,157$ & -76 \\
\hline Non-essential services closed & $-9,085$ & -4 \\
\hline Stay-at-home advisory & $-9,112$ & -31 \\
\hline Face coverings & $-9,109$ & -28 \\
\hline All interventions & $-9,617$ & -536 \\
\hline Full model (deaths) & $-4,096$ & NA \\
\hline Excluded & $-4,101$ & -5 \\
\hline Mass gathering restrictions & $-4,109$ & -13 \\
\hline Initial business closures & $-4,163$ & -66 \\
\hline Educational facilities closed & $-4,104$ & -8 \\
\hline Non-essential services closed & $-4,113$ & -17 \\
\hline Stay-at-home advisory & $-4,100$ & -4 \\
\hline Face coverings & $-4,569$ & -472 \\
\hline All interventions &
\end{tabular}

COVID-19: coronavirus disease; NA: not applicable.

increase household transmission. It could therefore not be surprising that stay-at-home measures on their own are not very effective outbreak control measures and may not generate large additional benefits.

There has been uncertainty about how beneficial the closing of educational establishments can be on coronavirus respiratory disease transmission [28,30-35], especially given that children often have mild or no symptoms [36]. We cannot resolve the lack of consensus in these lines of evidence about how likely children are to pass severe acute respiratory syndrome coronavirus 2 (SARS-CoV-2) to adults. Emergences of novel and seemingly more infectious variants [37] of the virus may complicate attempts to understand transmission patterns from children to adults using historical data and to understand the relative effectiveness of specific non-pharmaceutical interventions. Our study similarly does not identify which level of school closure has the most benefit, whether it is primary, junior, senior school or even higher education, although more recent evidence tends to point towards schooling between the ages of 11 and 19 years as being more likely to drive transmission than education for younger children [33]. Note that our own results are based on total closure rather than schools operating with at least partial social distancing. The impacts of partial school closures or social distancing controls within open schools need to be evaluated separately.
After closing educational establishments, the next greatest impact on the epidemiology of the European COVID-19 controls was from banning mass gatherings (which could be of any size), both public and private gatherings. A 2018 review of spread of respiratory infectious disease during mass gatherings found that most evidence was linked to the Islamic Hajj pilgrimage, where infections were mainly from rhinovirus, human coronaviruses and influenza A virus [38]. The evidence for respiratory disease outbreaks arising from other mass gatherings such as music festivals or sporting events is less established, but not absent. Several outbreaks of respiratory infectious disease have been linked to large festivals [38,39]. For instance, during the 2009/10 influenza season, pandemic influenza $\mathrm{A}\left(\mathrm{H}_{1} \mathrm{~N}_{1}\right)$ pdmog outbreaks were recorded at three of Europe's six largest music festivals, while some $40 \%$ of pandemic influenza cases that season in Serbia were linked to the Exit music festival. Analysis of COVID-19 NPI by other investigators using different approaches than ours also tend to find that banning large gatherings can be especially effective for reducing disease transmission [28].

The types of business closures are interesting. We established that there was weak collinearity between the two types of business closures in the models. However, the stronger association between a business closure control measure and case declines was with the initial business closures. Given that those initial closures were mostly directed at business where people congregate and that have a purpose of facilitating socialising (i.e. the hospitality industry), this would suggest that control measures among these businesses are where the most impact may be had. Although outbreaks of food poisoning are frequently linked with venues where food is consumed, this is much less frequent for outbreaks of respiratory infections. One exception was an outbreak of SARS at a restaurant where live palm civets were caged close to customer seating [40]. The link with COVID-19 is probably less about food and beverage consumption than about time people spend in close proximity to each other.

Similar to some other authors who have tried to assess relative importance of possible NPI in controlling COVID-19 and not found strong benefits for face-cover usage [41], we hesitate to interpret our findings on mask wearing as definitive. Mask advisories have not been implemented in isolation and were often implemented relatively late in the sequence of NPI in the group of European countries that we studied. Mask interventions were also implemented unevenly (as advisories or mandates) and usually only in limited settings. Our separate evidence review [42] found that mask wearing to stop respiratory disease transmission is likely to be only modestly effective, but we agree that when it comes to a pandemic situation, small protective measures may have cumulative important benefits [43]. 
Our study had limitations. Although our results suggests that closures of educational interventions and banning mass gatherings are the most important measures, this is caveated with several observations. Many interventions were implemented in different ways and at different points in the local epidemic. We relied on published and observed data which may have suffered from problems of under-ascertainment; the true effect of specific interventions may depend on true community prevalence that was not measured accurately enough. We did not undertake a systematic sensitivity analysis (excluding just one country per model, for instance) or adjustments in categorisations. It is likely that there will be serial dependency in the data as the level of disease at one time point is (inevitably) dependent on prior states of disease in the country, but we did not attempt to measure serial dependency in our models which might have further informed relative NPI efficacy. For example, in accordance with the IHME assignment, we treated Sweden as a country without school closures because schools for persons under 16 stayed open, although upper secondary and tertiary education facilities were actually shut in Sweden from late March 2020 [34]. Given recent evidence that secondary (age 11-19 years) rather than junior schools may play an important role in transmission of COVID-19, the educational closures in Sweden may explain in part the divergence from our predictions in that country [33]. Our models cannot allow for differences between countries regarding construction materials or ventilation rates in school buildings, which might influence transmissibility. The findings in support of school closures to contain the virus can truly only refer to schools when schools operate 'as normal' and not with COVID-19 mitigation practices in place. The exact timing of restrictions as reported by IHME being introduced varied over time in Italy, Spain and between individual federal states in Germany. Which types of work places could stay open varied; the acceptable reasons for being outdoors also varied between countries. Stay-at-home orders in some countries were an advisory but not enforced while elsewhere they were enforced by police with penalties. In some countries, children could go outside and outdoor exercise was permitted, while in others either or both might be banned. In some countries, severe travel restrictions were a separate intervention, while in others travel bans were a consequence of a stay-at-home order and could not be identified separately. Because of this variety in how interventions were implemented and described, the results for the potential of stay-athome advisories in particular may be underestimated. All models are simplifications of the complex nature of reality; our modelling was unable capture many subtle variations in how control measures were implemented. We acknowledge that lack of direct observation of these variations may have biased our results.

\section{Conclusion}

Relaxing stay-at-home orders and allowing reopening of non-essential businesses appeared to be the lowest risk measures to relax as part of plans to carefully lift COVID-19 lockdown measures. The pandemic started with little clear empirical evidence on the relative value of different interventions. Yet the reasons to implement only minimal control measures were compelling, given the social and economic harm linked to tight control measures. While we need to be cautious about using preliminary results, public health officials will have to use evidence as it emerges rather than wait for a final full view to decide what might be (was) the best control strategy. Careful monitoring of how relaxation of each control measure affects transmissibility of COVID-19 is required and will help to minimise the inevitably imperfect results.

\section{Acknowledgements}

Funding: Professor Hunter and Dr Brainard were funded by the National Institute for Health Research Health Protection Research Unit (NIHR HPRU) in Emergency Preparedness and Response at King's College London in partnership with Public Health England (PHE), in collaboration with the University of East Anglia. The views expressed are those of the author(s) and not necessarily those of the NHS, the NIHR, UEA, the Department of Health or Public Health England. Dr Colón-González is funded by the UK Space Agency Dengue Mosquito Simulation from Satellites (D-MOSS) project.

Data: The datasets used are in the public domain with copies available from the authors.

\section{Conflict of interest}

None declared.

\section{Authors' contributions}

PRH and JB conceived of the study. PRH and JB collected the data. PRH, FCG and SR undertook and refined analysis. PRH wrote the first draft which was revised by all authors. JB assembled revisions which all contributed to. Sharp-eyed reviewers and readers of the preprint contributed many small corrections via email.

\section{References}

1. Jacobs LA. Rights and quarantine during the SARS global health crisis: differentiated legal consciousness in Hong Kong, Shanghai, and Toronto. Law Soc Rev. 2007;41(3):511-52. https://doi.org/10.1111/j.1540-5893.2007.00313.x

2. Johnson HC, Gossner CM, Colzani E, Kinsman J, Alexakis L, Beauté J, et al. Potential scenarios for the progression of a COVID-19 epidemic in the European Union and the European Economic Area, March 2020. Euro Surveill. 2020;25(9):2000202. https://doi.org/10.2807/1560-7917. ES.2020.25.9.2000202 PMID: 32156332

3. Brooks SK, Webster RK, Smith LE, Woodland L, Wessely S, Greenberg N, et al. The psychological impact of quarantine and how to reduce it: rapid review of the evidence. Lancet. 2020;395(10227):912-20. https://doi.org/10.1016/S01406736(20)30460-8 PMID: 32112714

4. Office for Budget Responsibility (OBR). Coronavirus lockdown to deliver large (but hopefully temporary) shock to the economy and public finances. London: OBR; 2020. Available from: https://obr.uk/category/coronavirus

5. Massaro E, Ganin A, Perra N, Linkov I, Vespignani A. Resilience management during large-scale epidemic outbreaks. Sci Rep. 
2018;8(1):1859. https://doi.org/10.1038/s41598-018-19706-2 PMID: 29382870

6. Adam D. Special report: The simulations driving the world's response to COVID-19. Nature. 2020;580(7803):316-8. https:// doi.org/10.1038/d41586-020-01003-6 PMID: 32242115

7. Sridhar D, Majumder MS. Modelling the pandemic. BMJ. 2020;369:m1567. https://doi.org/10.1136/bmj.m1567 PMID: 32317328

8. Borenstein S, Johnson CK. Modeling coronavirus: 'Uncertainty is the only certainty'. New York: Associated Press; 2020. Available from: https://apnews.com/88866498ff5c908e5f28f7 b5 5 e5b695

9. Andrews C. Predicting the pandemic: mathematical modelling tackles Covid-19. London: Engineering and Technology; 2020. Available from: https://eandt.theiet.org/content/ articles/2020/04/predicting-the-pandemic-mathematicalmodelling-tackles-covid-19

10. McCoy D. Faith in coronavirus modelling is no substitute for sound political judgment. London: The Guardian; 2020. Available from: https://www. theguardian.com/commentisfree/2020/apr/10/ modelling-pandemic-politicians-decisions-science

11. Rosenbaum PR. How to see more in observational studies: Some new quasi-experimental devices. Annu Rev Stat Appl. 2015;2(1):21-48. https://doi.org/10.1146/ annurev-statistics-010814-020201

12. Harris AD, McGregor JC, Perencevich EN, Furuno JP, Zhu J, Peterson DE, et al. The use and interpretation of quasiexperimental studies in medical informatics. J Am Med Inform Assoc. 2006;13(1):16-23. https://doi.org/10.1197/jamia.M1749 PMID: 16221933

13. Besag J, York J, Mollié A. Bayesian image restoration, with two applications in spatial statistics. Ann Inst Stat Math. 1991;43(1):1-20. https://doi.org/10.1007/BFo0116466

14. Obaromi D. Spatial modelling of some conditional autoregressive priors in a disease mapping model: the Bayesian approach. BJSTR. 2019;14(3):10680-6. https://doi. org/http://dx.doi.org/10.26717/BJSTR.2019.14.002555

15. Bi Q, Wu Y, Mei S, Ye C, Zou X, Zhang Z, et al. Epidemiology and transmission of COVID-19 in 391 cases and 1286 of thei close contacts in Shenzhen, China: a retrospective cohort study. Lancet Infect Dis. 2020;20(8):911-9. https://doi. org/10.1016/S1473-3099(20)30287-5 PMID: 32353347

16. Backer JA, Klinkenberg D, Wallinga J. Incubation period of 2019 novel coronavirus (2019-nCoV) infections among travellers from Wuhan, China, 20-28 January 2020. Euro Surveill. 2020;25(5):2000062. https://doi.org/10.2807/1560-7917. ES.2020.25.5.2000062 PMID: 32046819

17. Lauer SA, Grantz KH, Bi Q, Jones FK, Zheng Q, Meredith HR, et al. The incubation period of coronavirus disease 2019 (COVID-19) from publicly reported confirmed cases: estimation and application. Ann Intern Med. 2020;172(9):577-82. https:// doi.org/10.7326/M20-0504 PMID: 32150748

18. Elias C, Sekri A, Leblanc P, Cucherat M, Vanhems P. The incubation period of COVID-19: A meta-analysis. Int J Infect Dis. 2021;104:708-10. https://doi.org/10.1016/j.ijid.2021.01.069 PMID: 33548553

19. Docherty $A B$, Harrison EM, Green CA, Hardwick HE, Pius $R$, Norman L, et al. Features of 16,749 hospitalised UK patients with COVID-19 using the ISARIC WHO Clinical Characterisation Protocol; medRxiv 2020. https://doi. org/10.1101/2020.04.23.20076042

20. Zhou F, Yu T, Du R, Fan G, Liu Y, Liu Z, et al. Clinical course and risk factors for mortality of adult inpatients with COVID-19 in Wuhan, China: a retrospective cohort study. Lancet. 2020;395(10229):1054-62. https://doi.org/10.1016/S01406736(20)30566-3 PMID: 32171076

21. Verity R, Okell LC, Dorigatti I, Winskill P, Whittaker C, Imai N, et al. Estimates of the severity of coronavirus disease 2019: a model-based analysis. Lancet Infect Dis. 2020;20(6):66977. https://doi.org/10.1016/S1473-3099(20)30243-7 PMID: 32240634

22. Chatterjee S, Hadi AS. Regression analysis by example. 4th ed. Hoboken: John Wiley \& Sons; 2015.

23. Regorz A. How to interpret a collinearity diagnostics table in SPSS. Regorz Statiztik; 2020. Available from: http://www. regorz-statistik.de/en/collinearity_diagnostics_table_SPSS. html

24. Wright O. People told to wait ten days for coronavirus test results. London: The Times; 2020. Available from: https:// www.thetimes.co.uk/article/people-told-to-wait-ten-days-forcoronavirus-test-results-7dpjbot96

25. Pan A, Liu L, Wang C, Guo H, Hao X, Wang Q, et al. Association of public health interventions with the epidemiology of the COVID-19 outbreak in Wuhan, China. JAMA.
2020;323(19):1915-23. https://doi.org/10.1001/jama.2020.6130 PMID: 32275295

26. Zhang J, Litvinova M, Liang Y, Wang Y, Wang W, Zhao S, et al. Changes in contact patterns shape the dynamics of the COVID-19 outbreak in China. Science. 2020;368(6498):1481-6. https://doi.org/10.1126/science.abb8001 PMID: 32350060

27. Zhang J, Litvinova M, Wang W, Wang Y, Deng X, Chen X, et al. Evolving epidemiology and transmission dynamics of coronavirus disease 2019 outside Hubei province, China: a descriptive and modelling study. Lancet Infect Dis. 2020;20(7):793-802. https://doi.org/10.1016/S14733099(20)30230-9 PMID: 32247326

28. Haug N, Geyrhofer L, Londei A, Dervic E, Desvars-Larrive A, Loreto V, et al. Ranking the effectiveness of worldwide COVID-19 government interventions. Nat Hum Behav. 2020;4(12):1303-12. https://doi.org/10.1038/s41562-02001009-0 PMID: 33199859

29. Koh WC, Naing L, Chaw L, Rosledzana MA, Alikhan MF, Jamaludin SA, et al. What do we know about SARS-CoV-2 transmission? A systematic review and meta-analysis of the secondary attack rate and associated risk factors. PLoS One. 2020;15(10):e0240205. https://doi.org/10.1371/journal. pone.0240205 PMID: 33031427

30. Viner RM, Russell SJ, Croker H, Packer J, Ward J, Stansfield $C$, et al. School closure and management practices during coronavirus outbreaks including COVID-19: a rapid systematic review. Lancet Child Adolesc Health. 2020;4(5):397-404. https://doi.org/10.1016/S2352-4642(20)30095-X PMID: 32272089

31. Cowling BJ, Lau EH, Lam CL, Cheng CK, Kovar J, Chan KH, et al. Effects of school closures, 2008 winter influenza season, Hong Kong. Emerg Infect Dis. 2008;14(10):1660-2. https://doi. org/10.3201/eid1410.080646 PMID: 18826841

32. Hens N, Ayele GM, Goeyvaerts N, Aerts M, Mossong J, Edmunds JW, et al. Estimating the impact of school closure on social mixing behaviour and the transmission of close contact infections in eight European countries. BMC Infect Dis. 2009;9(1):187. https://doi.org/10.1186/1471-2334-9-187 PMID: 19943919

33. Flasche S, Edmunds WJ. The role of schools and school-aged children in SARS-CoV-2 transmission. Lancet Infect Dis. 2021;21(3):298-9. PMID: 33306982

34. Folkhälsomyndigheten [Public Health Agency of Sweden]. COVID-19 in schoolchildren: A comparison between Finland and Sweden. Stockholm: Public Health Agency of Sweden; 2020. Available from: www.folkhalsomyndigheten.se/contentassets/ c1b78bffbfde4a7899ebod8ffdb57bo9/covid-19-school-agedchildren.pdf

35. von Bismarck-Osten C, Borusyak K, Schönberg U. The role of schools in transmission of the SARS-CoV-2 virus: Quasiexperimental evidence from Germany. Essen: Ruhr Economic Papers; 2020. Available from: https://www.rwi-essen.de/ media/content/pages/publikationen/ruhr-economic-papers/ rep_20_882.pdf

36. Shen K, Yang Y, Wang T, Zhao D, Jiang Y, Jin R, et al. Diagnosis, treatment, and prevention of 2019 novel coronavirus infection in children: experts' consensus statement. World J Pediatr. 2020;16(3):223-31. https://doi.org/10.1007/s12519-02000343-7 PMID: 32034659

37. Public Health England (PHE). Investigation of novel SARSCOV-2 variant: variant of concern 202012/01. London: PHE; 2020. Available from: https://www.gov.uk/government/ publications/investigation-of-novel-sars-cov-2-variant-variantof-concern-20201201

38. Hoang V-T, Gautret P. Infectious diseases and mass gatherings. Curr Infect Dis Rep. 2018;20(11):44. https://doi.org/10.1007/ S11908-018-0650-9 PMID: 30155747

39. Botelho-Nevers E, Gautret P. Outbreaks associated to large open air festivals, including music festivals, 1980 to 2012. Euro Surveill. 2013;18(11):20426. https://doi.org/10.2807/ ese.18.11.20426-en PMID: 23517872

40. Wang M, Yan M, Xu H, Liang W, Kan B, Zheng B, et al. SARS$\mathrm{CoV}$ infection in a restaurant from palm civet. Emerg Infect Dis. 2005;11(12):1860-5. https://doi.org/10.3201/eid1112.041293 PMID: 16485471

41. Brauner JM, Mindermann S, Sharma M, Johnston D, Salvatier J, Gavenčiak T, et al. Inferring the effectiveness of government interventions against COVID-19. Science. 2021;371(6531):eabd9338. PMID: 33323424

42. Brainard J, Jones NR, Lake IR, Hooper L, Hunter PR. Community use of face masks and similar barriers to prevent respiratory illness such as COVID-19: a rapid scoping review. Euro Surveill. 2020;25(49):2000725. https://doi.org/10.2807/1560-7917. ES.2020.25.49.2000725 PMID: 33303066

43. Cowling BJ, Leung GM. Face masks and COVID-19: don't let perfect be the enemy of good. Euro Surveill. 
2020;25(49):2001998. https://doi.org/10.2807/1560-7917.

ES.2020.25.49.2001998 PMID: 33303063

44. Watanabe S. Asymptotic equivalence of Bayes cross validation and widely applicable information criterion in singular learning theory. J Mach Learn Res. 2010;11(Dec):3571-94.

45. Pettit L. The conditional predictive ordinate for the normal distribution. J R Stat Soc B. 1990;52(1):175-84. https://doi. org/10.1111/j.2517-6161.1990.tb01780.x

\section{License, supplementary material and copyright}

This is an open-access article distributed under the terms of the Creative Commons Attribution (CC BY 4.0) Licence. You may share and adapt the material, but must give appropriate credit to the source, provide a link to the licence and indicate if changes were made.

Any supplementary material referenced in the article can be found in the online version.

This article is copyright of the authors or their affiliated institutions, 2021. 